\title{
The role of gene architecture in gene expression
}

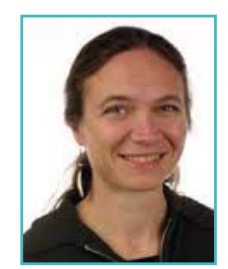

My lab is interested in the interplay between transcription and splicing. Recent global studies in several species have indicated that most intron removal is co-transcriptional, underscoring the potential for coupling between the transcription and splicing machineries as well as chromatin. Indeed, we recently discovered Terminal Exon Pausing, in which transcription elongation slows in short last exons of genes in budding yeast (Carrillo Oesterreich et al., 2010). The effect of TEP is to ensure that pre-mRNA splicing is completed before transcription termination. We have further pursued the role of gene architecture in transcription and processing, showing that short first exons act as transcriptional enhancers (Bieberstein et al., 2012). In addition, we study RNA-protein inter- actions and their functions (SR proteins and the cap binding complex) as well as the structure and function of RNA rich cellular compartments, such as Cajal bodies. Our work is conducted in mammalian tissue culture cells, zebrafish embryos, and budding and fission yeasts. On this occasion, I will report on a new study investigating the role of gene architecture in the activation of transcriptional programs in zebrafish embryos.

\section{References:}

Bieberstein NI, Carrillo Oesterreich F, Straube K, Neugebauer KM. (2012) First exon length controls active chromatin signatures and transcription. Cell Rep. 2(1), 62-68.

Carrillo Oesterreich F, Preibisch S, Neugebauer KM. (2010) Global analysis of nascent RNA reveals transcriptional pausing in terminal exons. Mol Cell. 40(4), 571-81. 